\title{
GeoHealth: a location-based service for home healthcare workers
}

\author{
Jesper Kjeldskov*, Claus M. Christensen and Klaus K. Rasmussen \\ Department of Computer Science, Aalborg University, Aalborg, Denmark
}

(Received 11 May 2008; final version received 29 January 2010; accepted 2 March 2010)

\begin{abstract}
We describe a map-based location-based service 'GeoHealth' for home healthcare workers who attend patients at home within a large geographical area. Informed by field studies of work activities and interviews with care providers, we have designed a mobile location-based service prototype supporting collaboration through information sharing and distributed electronic patient records. The GeoHealth prototype gives the users live contextual information about patients, coworkers, current and scheduled work activities and alarms adapted to their geographical location. The application is web-based and uses Google Maps, Global Positioning System (GPS) and Web 2.0 technology to provide a lightweight, dynamic and interactive representation of the work domain supporting distributed collaboration, communication and peripheral awareness among nomadic workers. Through a user-based evaluation, we found that the healthcare workers were positive towards the use of location-based services in their work, and that the dynamic and interactive geospatial representation of the work domain provided by GeoHealth supported distributed collaboration, communication and peripheral awareness. We also identified areas for improvements.
\end{abstract}

Keywords: map-based LBS; LBS interaction design; LBS user experience; Web 2.0, Ajax; home healthcare; mobile healthcare applications; field study

\section{Introduction}

Location-based services (LBSs) constitute an emerging paradigm of computing where users on the move are provided with information and functionality that is particularly relevant at a specific geographical location or within a specific distance. Within this broad scope, many systems have been developed and described in the research literature over the past decade, envisaging 'blue sky' innovations, exploring user experiences and tackling technical challenges of implementation (Raper et al. 2007). The research field of LBSs has developed significantly in its relatively short lifetime and core technologies are now maturing for deployment outside the scope of research prototypes. Facilitated by this, increasing research is reported into the user side of the equation exploring new potential 'real-world' application areas and studying the use of LBSs in these settings. This is, indeed, an interesting time to do LBS research.

*Corresponding author. Email: jesper@cs.aau.dk 
As a specific case for our own research within the area of LBS development and use, we have studied work activities in regional home healthcare as a potential application area of this emerging technology. Home healthcare is a subset of the healthcare domain where patients are treated or attended to in their own homes rather than at a hospital or a clinic. Like in most areas of the healthcare domain, home healthcare workers are under increasing pressure to provide improved care to more people while using fewer resources. At the same time, it is often speculated that ubiquitous and mobile computing may provide some means for improving quality of service through, for example, better communication between patients and healthcare workers, by providing access to centralised medical data at the point of care, supporting effective emergency response, etc. In response to this, we have been exploring the development and use of a location-based mobile healthcare system supporting the nomadic collaborative work activities of physically distributed home healthcare workers by providing mobile and location-based access to electronic patient records as well as information about coworkers and means for communication and shared work task management.

Recent developments in web-based technologies and services, availability of GPS positioning on mobile and portable devices and widespread access to broadband mobile Internet have enabled the creation of a new class of LBSs. Combining positioning capabilities with the powers of 'Web 2.0' technologies for user-generated content, open application programming interfaces (APIs) for geographical interfaces such as Google Maps and mobile or portable devices with full-blooded web browsers now makes it easier than ever to implement LBSs and applications for users on the move that take into consideration their location and the surrounding environment. Hence, Raper et al. (2007, p. 29) report 'an explosion of activity and interest' in using Web 2.0 technologies for the development of 'the next wave' of LBSs.

Inspired by these new technological possibilities, our latest location-based prototype application 'GeoHealth' represents an early instance of this potential next wave of Web 2.0 based LBSs described by Raper et al. (2007). GeoHealth is a web-based LBS running within the web browser on a laptop or tablet PC. It graphically augments a home healthcare district with a meshup of context information (e.g. about patients, coworkers, current and scheduled work activities and alarms), user-generated information (e.g. text messages and treatment notes) and information stored centrally (e.g. medical patient records and work schedules). The aims and objectives of the GeoHealth prototype were to explore the user experience of a mobile LBS within the healthcare domain exploring the powers of Web 2.0 technologies in combination with GPS positioning a geographical interface, and the mobile Internet.

Reporting on this work in the Journal of Location Based Services, we contribute to two of the 'urgently needed' areas of research outlined in Raper et al.'s (2007) editorial lead paper of the first issue of the journal: interaction design for LBS and LBS architectures and platforms. We also respond to the lead paper's recommendation for more user-centred system conception of LBSs (Raper et al. 2007, p. 22).

This article is structured as follows. First, we present related work within context-awareness, LBSs and mobile healthcare applications. Second, we introduce our case of home healthcare workers. Third, we present the GeoHealth prototype followed by findings from a user-based evaluation. Finally, we conclude on our research and point out avenues for further work. 


\section{Related work}

In this section, we outline the related work within context-awareness, LBSs and mobile healthcare applications.

\subsection{Context awareness and LBSs}

There has been a lot of research on context-awareness in recent years. Much of this has enabled computers to collect and formalise information about contextual factors describing, for example, the geographical, environmental, biometrical, and social setting and stage of a user. In parallel, this has inspired the use of machine intelligence to make mobile systems transform themselves accordingly and minimise need for explicit user interaction. Experiences with developing and using such systems are many and context-awareness has proven possible within specialised domains such as industrial process control, healthcare, transportation and tourism (Cheverst et al. 2000, Bardram 2004, Skov and Høegh 2006, Paay et al. 2009). However, research has also identified many fundamental problems with this approach to human-computer interaction. Even simple adaptations to context, such as the mobile phone that knows when not to ring, are extremely complex to implement, and any context-aware technology is most likely to make mistakes and take control away from the user (Barkhuus and Dey 2003, Brown and Randell 2004). In contrast, it has been stressed that while computational systems are good at gathering and aggregating data, humans are good at noticing, integrating and interpreting obvious as well as subtle cues and determining appropriate actions (Kjeldskov and Paay 2006). In response, some researchers have advocated that another way to deal with complex and ambiguous context information is to actively involve the user (Dey and Mankoff 2005) and make humans, not machines, the consumers of context information by representing it in the interface rather than automatically adapting to it (Aaltonen and Lehikoinen 2005, Edwards 2005). As a fictive example, a representational context-aware service for a hospital ward would display collected context-information about patients, healthcare workers and current work activities, but leave interpretation and decision making on the basis of this information to the user rather than to the computer.

Real examples of context-aware systems embodying this representational approach are still very few and mostly research prototypes rather than commercial systems. For example, Paulos and Goodman's (2004) jabberwocky prototype use detected patterns of nearby Bluetooth devices over time for visualising 'familiar strangers' in the users' vicinity. Erickson et al.'s (2002) Babble prototype visualise 'social proxies' based on the presence and activities of nearby people. In a more recent study, Lemmelä and Korhonen (2007) use geographical context information, gathered from salient keywords within public 'geo-postings', to visualise physical clusters of similar places on a digital map, such as areas with a high occurrence of the key word 'restaurant', 'shopping', 'art' or 'museum'. Common for these systems is that they are context-aware but use their awareness to generate new information rather than trying to reduce it. It is also common that they are particularly aware of their location.

One of the most promising aspects of user context for a mobile or portable computer system to respond to is location (Kaasinen 2003, Jones et al. 2004, 
Raper et al. 2007). As a subset of context-aware systems, LBSs represent an emerging class of computer systems providing mobile device users with information and functionality that is particularly relevant at a specific geographical location or within a specific distance. Within the last decade, this class of mobile or portable computer systems has received increasing attention from researchers within areas such as computer science, human-computer interaction and interaction design, as well as from software industry. Commercially available LBSs are still relatively few, but an increasing number of services are now emerging that integrate wide-area broadband wireless Internet access, web resources and geographical information for personal digital assistants (PDAs) and 'smart phones' with GPS and other positioning capabilities. As pointed out in Raper et al.'s (2007, p. 6) opening article of the first issue of the Journal of Location Based Services, one of the reasons for the less than expected user take-up of LBSs is that many of the potential application areas for useful LBSs have simply not yet been explored by mobile operators. One of these underexplored domains is healthcare. Within the healthcare domain, geographical positioning technologies have a history of use in emergency response systems as a means of pinpointing people in distress and directing information to relevant personnel. However, the use of $L B S s$ as such is still a rarity despite the huge potentials of, for example, relating the vast amount of available digital information to geographical locations in the creation of new and effective medical services.

Yet, developing successful LBSs for a potential application domain such as healthcare is not trivial either. It inherits challenges of context-awareness and other LBSs described in the literature, such as issues of user control, privacy and determining people's location in physical space, as well as introducing new ones. Little is known about people's use of such of services, how to design them well and what is (and is not) useful. It is unknown how users perceive and use information provided through an LBS, what content is considered relevant (and what is not), and how people will adopt and appropriate information services that react to their location and combine, for example, web content, satellite imaging and cartography. Hence, more research is needed into the development and real-world use of LBSs. Through the case study presented below, our aim is to contribute to this area with (1) understanding of a specific potential use domain for LBSs and technologies,

(2) concrete design ideas for LBS for home healthcare workers and (3) a Web 2.0 approach to the technical implementation LBSs for exploring latest trends in web-based technology and geographical information systems.

\subsection{Mobile healthcare applications and LBSs}

Mobile healthcare applications is an evolving area within health informatics where recent advances in ubiquitous and mobile computing are exploited for the creation of new, flexible and effective medical services (Woodward et al. 2001, Tachakra et al. 2003, Varshney 2003). The area covers a wide range of healthcare applications and services where patients and/or healthcare workers are free to move around physically, while still connected to the service (for a review of systems, see e.g. Pattichis et al. 2002). One of the most prevalent mobile healthcare applications reported in the literature is the use of biometrical sensors and mobile phones for monitoring patient data by oneself (Mattila et al. 2008) or remotely in real time 
(Tachakra et al. 2003, Figueredo and Dias 2004) allowing, for example, non-critical patients to remain at home as much as possible. Other applications involve the use of high-bandwidth mobile data networks for live transmission of video data from, for example, ambulances on dispatch to health professionals at the hospital (e.g. Mandellos et al. 2004), pervasive access to electronic patient data for healthcare workers on the move (e.g. Reponen et al. 2000) and patient telemonitoring in emergency situations (Maglogiannis and Hadjiefthymiades 2007, Maglogiannis et al. 2009). Some mobile healthcare applications also support 'telecooperation' amongst physically distributed and mobile healthcare workers through information sharing, team communication and coordination of team activities via mobile terminals and wireless data networks (e.g. Pitsillides et al. 1999).

As a specific area within healthcare applications, LBSs and interactive map applications have received increasing attention within the last couple of years. In Boulos (2003b), location-based health services are promoted as a new paradigm for personalised information delivery responding to the potential information overload potentially created by unfiltered delivery of health information to various mobile devices. The main goal of this paradigm is to create better presentation of health and healthcare needs and Internet resources across a geographical area in order to facilitate better support for decision making. As a specific example of such LBS, 'HealthCyberMap' is an interactive geographical map for browsing medicine and health information on the Internet (Boulos 2003a). Enabling location-based browsing of health information and collection of spatial data, Rainham et al. (2008) presents the design and evaluation of a wearable GPS system and data logger developed to assist in deriving a more complete picture of the different places that influence people's health and well-being. Using similar technologies, Boulos et al. (2007) describe a location-based healthcare service aimed at increasing older people's autonomy through the use of a wearable monitoring device with GPS connected to a central care service and monitoring system. Exploring the use of LBSs as a part of a ubiquitous computing environment at a large hospital, Kjeldskov and Skov (2007) presented findings on the usability of the 'MobileWard' system giving nurses and doctors access to electronic patient records and work tasks based on their physical location.

In a user-based study of requirements for geographic information system (GIS)-based decision support in public health, Driedger et al. (2007) found that maps and mapping tools were powerful means of "converting locally collected data into information' and that desired functionality for such systems included the ability to geo-code patient data and push-pin patients and services on a shared map base of the region. Extending the use of such shared map-based representations, Chang et al. (2009) presented a combined Google Earth and GIS mapping system designed to assist managing of dengue fever in a developing country. This system makes use of several interactive information layers overlaid onto a map of a specific region, allowing public health workers to collaboratively interpret spatial relationships between data. Most of the applications reviewed here use GPS as the primary positioning technology. For a detailed review of technologies for detecting user location in healthcare, see Boulos (2003b).

In the study presented in this article, we focus on a combination of technologies where a map-based location-based service provides the foundation for supporting GPS tracked home healthcare workers in their work activities through mobile assess 
to electronic patient record data, channels for group communication, tools for coordination and simple patient monitoring.

\section{Field study: home healthcare}

Home healthcare is a subset within the healthcare domain where patients are treated or attended to in their own homes rather than at a hospital or a clinic. Home healthcare nursing and assistance is mostly used for providing care to elderly people, or people with disabilities, who with this nursing or assistance are then capable of taking care of themselves in their everyday lives. It is also often used as a means of follow-up treatment subsequent to periods of hospitalisation. Making house calls to patients at home obviously requires nurses and healthcare assistants to be mobile within a specific geographical area 'nomadically' (Sawhney and Schmandt 2000) moving their location of work from place to place throughout the day. It also requires them to be physically distributed from their coworkers, while still collaborating on shared tasks coordinated through division of labour, shared plans and ongoing communication.

With the purpose of informing the design of an LBS for nomadic home healthcare workers, we conducted an empirical field study of work activities in the municipality of Aars in Denmark in 2006. The study was done using a rapid ethnography approach (Millen 2000) involving a combination of observations of current practice, contextual interviews and semi-structured interviews. The study involved 10 healthcare assistants and 11 nurses with different levels of experience with the work domain, and resulted in $17.5 \mathrm{~h}$ of audio data accompanied with field notes and digital photographs. Findings from the study were verified through subsequent meetings and focus groups with the home healthcare staff.

The focus of the study was to enquire into the characteristics of the distributed work activities and the relationships between work activities, patients, healthcare workers, information, information needs and geographical space. For example, we were interested in knowing who needs what information when and where, how division of labour is negotiated, assigned, represented and managed, how individual workdays are structured (and restructured) in relation to geographical space, what happens in case of events out of the ordinary, what information is kept as a permanent record for later use, what communication goes on between the distributed workers throughout the day, etc. Furthermore, we wanted to identify specific opportunities for supporting work activities, collaboration and communication by means of a LBS representing key contextual data in a geographical interface.

Data from the field study were analysed through a process of grounded coding and affinity diagramming of higher-level themes (Strauss 1987, Beyer and Holtzblatt 1998). On the basis of the field data, we also created four different personas (Pruitt and Adlin 2006) describing key characteristics of prospective users, their work activities, goals and habits with the purpose of serving as hypothetical archetype users for the design team. Finally, we created a number of physical models (Beyer and Holtzblatt 1998) of the workspaces of nurses and healthcare assistants illustrating how they operate during a typical workday in the municipality. 


\subsection{Findings from field study}

In this section, we present a concentrate of findings from our find studies. These findings serve as a foundation for our prototype design, but also illustrate the type of data, insight and richness that is typically gained from this methodological approach within the field of human-computer interaction. In conjunction with our prototype system, we wish to illustrate how this field study approach is also of value in relation to design and development of LBSs.

Home healthcare in the observed municipality involves two types of workers: nurses and healthcare assistants. The nurses have overall responsibility for the treatment of patients and also administer medicine to patients at their homes. The healthcare assistants are responsible for daily treatment and serve as 'daily observers' reporting back to the nurses in case of, for example, side effects from medication. Currently, the nurses and healthcare assistants use a series of paper-based information artefacts and mobile and landline phones for coordinating and communicating with each other throughout the day and between shifts.

The healthcare assistants have a pre-planned workday with schedules for visiting assigned patients at particular times and in a particular order.

The division of work assignments happens in central meetings, but is also altered by the healthcare assistants during a day if something out of the ordinary comes up. Supplementing the day schedule, they carry specific task lists for the caretaking of each patient including, for example, shopping lists for groceries. In addition to this, they carry a paper notebook used to jot down needs for changes to the services or schedule, which are then entered in to the permanent record at the central office by a manager after the shift. Finally, the healthcare assistants carry sheets of paper for documenting mileage, which is used for compensating the use of private cars for work purposes.

The nurses' workdays are more ad hoc than the healthcare assistants'. Rather than a pre-planned schedule of visits, each nurse carry reference sheets for all his or her assigned patients in the district. These sheets contain personal information, phone numbers for the general doctor or practitioner, overview of health conditions, remarks on treatment and things to be aware of, diagnosis, phone numbers for relatives, lists of nursing tasks and practical information such as the location of the key in the patients' homes. They also indicate what time the patients are usually visited by nurses or healthcare assistants during the week. Every morning, the nurses look through their reference sheets, pick out the patients they are supposed to visit that day and manually transfer this information to the corresponding page in their calendar. However, rather than making a precise time schedule for the day, the 'day-view' functions more as a loosely scheduled 'to-do list' with a few notes. In addition to their reference sheets and calendars, the nurses carry paper notebooks in which they register changes to the treatment and/or medication of patients, which they then enter in to the permanent record later at the central office. Finally, the nurses also register how many kilometres they drive in their private car for work purposes for economical compensation.

In order to facilitate collaboration, the healthcare assistants and nurses share a series of paper-based records located either at the individual patients or at the central office. These records function partly as repositories of information on treatment for documentation purposes, and partly as media for communication between workers 
and between shifts. First, each patient has an individual paper-based 'book' located at his or her home and used for general 'all-to-all' information and communication between healthcare workers, the patient and their relatives. In addition to this, medicated patients have a paper-based medical prescription located with their medicine. Second, the healthcare assistant and nurses collaborate and communicate through paper-based records at the central office containing more detailed information not suitable for public view (i.e. relatives). Third, the nurses collaborate through the official medical records, which are increasingly in electronic form.

In addition to coordinating their collaborative efforts through shared written notes and records, the nurses and healthcare assistants make extensive use of direct synchronous communication through mobile and landline phones. All nurses are equipped with mobile phones, making them immediately reachable in case of an emergency situation. The healthcare assistants do not have mobile phones but communicate by means of their patients' landline phones, and thus have to estimate the whereabouts of each other before making calls. Three kinds of synchronous communication were observed. First, coworkers (healthcare assistants, nurses, physiotherapists, janitors, staff at the central kitchen, etc.) are informed about progress or delays in their work schedule, which may influence the work assignments of others. This could be, for example, when a healthcare assistant has finished bathing a patient, who is then ready for the nurse. It could also be in the event that a patient is suddenly hospitalised and thus does not need food delivery or assistance at home. Second, the healthcare assistants sometimes call each other to negotiate ad hoc changes of work tasks in the case of something out-of-the ordinary happening. Third, and perhaps most importantly, the healthcare workers communicate orally in emergency situations, which are usually triggered by patient alarms. When a patient triggers the alarm button (situated centrally in their home), the alarm is directed to a dedicated 'emergency phone' carried by one of the healthcare assistants. This person is then responsible for delegating dispatch to the closest healthcare worker. This is done by making a series of phone calls from the closest landline; first to the place of the alarm in order to assess the situation, and then to the colleagues who is believed to be closest to the patient in need. Eventually, a worker is dispatched to the alarm.

From our field studies, it is clear that current work practice have enablers as well as challenges for providing optimal care. On the positive side, the lose mechanisms for ad hoc exchange of work tasks makes it easy for the workers to respond to events out-of-the ordinary. Access to shared repositories of information makes it possible to coordinate treatment among coworkers, and communicate relatively easily between shifts. Finally, self-structuring of the workday allows for flexibility and for assisting each other if needed. First on the negative side, the use of paper-based notes and records requires double registration of information and results in a delay between time of registration and time of availability to coworkers. Having several parallel records with overlapping content also introduces redundancy and uncertainty about what information is the newest. Second, relying on non-mobile technology for communication between mobile workers introduces inefficiency and risks. Important messages may not reach their recipients in time or may never be communicated in the first place because of doubt about where to call. In terms of alarms, the risk of losing valuable time in the process of locating and dispatching the closest healthcare assistant or nurse is obvious. 
As a case for LBSs, the field study reveals a number of properties, which makes this a potentially interesting application area. Home healthcare workers are highly mobile and geographically distributed throughout a workday, and their work tasks are highly tied to specific geographical locations. Not all information is relevant at all times of the workday, but can to a large extent be coupled to specific locations that the healthcare workers will visit during the day. Hence, location could be used as a powerful filtering parameter for this particular work domain, and the functionality needed by home healthcare workers could be considered and presented as location-based information services. In addition to this, the dynamic locations of coworkers in relation to one self constitute a central factor for ad hoc management of situations out-of-the ordinary, and could also be considered an LBS candidate.

\section{The GeoHealth prototype}

Informed by our field study, we designed and implemented a functional prototype, GeoHealth, which supports distributed and mobile collaboration through representation of live contextual information about patients, coworkers, current and scheduled work activities and alarms adapted to the user's location (Figure 1).



Figure 1. The main screen of the GeoHealth prototype system with the layers of four healthcare workers' patients, routes and current location enabled. In the palette on the right, the user can see her own scheduled work tasks and keep an eye on which colleague is closest to her. Using the timeline below the map, the user can 'fast forward' into the future and see how the schedules for the day will unfold according to plan, or 'rewind into the past' to see where people have already been today. 
Using the terminology of Raper et al. (2007, p. 20), GeoHealth is a 'map-based location based service.'

The prototype explores new technical opportunities for the creation of multiuser location-based web-applications through a combination of Web 2.0 technology, GPS positioning, interactive map/satellite image overlays and mobile Internet access. GeoHealth was purposely designed for a sub-notebook size laptop or tablet computer that the healthcare workers can easily carry with them in a small bag. We deliberately refrained from designing for a smaller mobile form factor such as PDA handheld devices because of the limitations of these in terms of screen size. We have previously designed and evaluated other systems for healthcare using context-aware handheld devices (Kjeldskov and Skov 2007), and found from empirical studies that this form factor was often too limiting for healthcare workers, and that a small laptop or tablet was often a more suitable form factor for this kind of work. Aiming at a small laptop or tablet also allowed us to design GeoHealth to integrate well with other relevant applications (such as Skype), and to fully explore the use of Web 2.0 functionality. Based on findings from our field study, the GeoHealth prototype was developed through a semi-structured iterative process for moving from ethnographic data towards user interface design, involving activities of design sketching, paper prototyping and technology exploration (Paay 2008).

The basic idea behind the GeoHealth system was to provide the home healthcare workers with an interactive graphical representation of their shared and individual work domains on a small laptop or tablet computer. This representation should be embedded into a digital map of their physical surroundings in different layers (Figure 2). Within this representation, the workers should be able to directly access

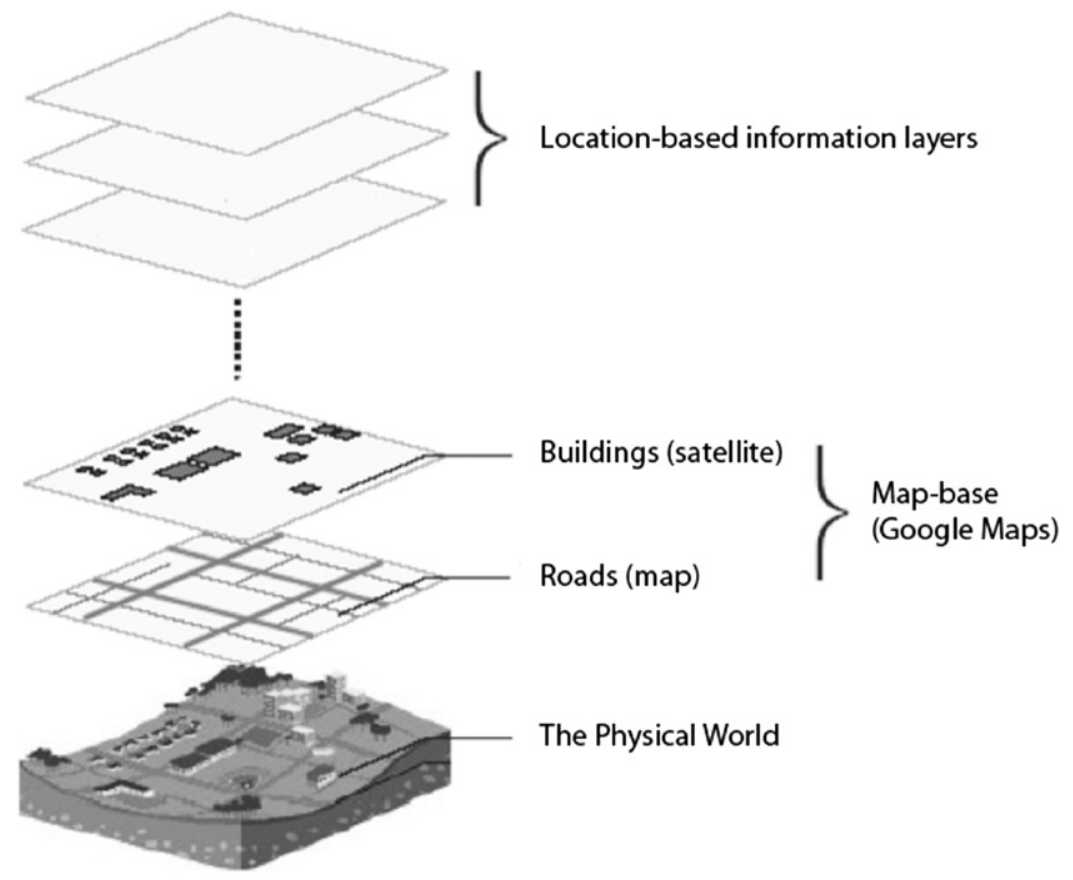

Figure 2. The geographical information-layering model of GeoHealth. 
key information about their patients, scheduled tasks and the location and activities of their colleagues. At the same time, the system should be able to function as a medium for text-based messaging, facilitate easy ad hoc exchange of work tasks and it should function as a graphical interface to the existing patient alarm system replacing needs for making phone calls. We also wanted to facilitate spoken communication among the workers through voice over Internet protocol (VoIP), as well as automate mileage registration for private car use.

We intentionally made the system context-aware in the sense that it would know the whereabouts and activities of its users, the patients and scheduled work assignments. However, rather than using this information to make the system 'intelligently' suggest to the users what to do, we wanted to represent context information in the interface and leave the intelligence to the users. Hence, GeoHealth deploys active context-awareness (Barkhuus and Dey 2003) in the sense that it pushes information to the user when, for example, he or she enters the vicinity of an assigned patient, or if a patient alarm is triggered nearby. However, leaving the user in control, the system also deploys passive context-awareness in the sense that pushed context information appears discreetly within the interface, and goes away again automatically if the user does not act on it. We call this combination of active and passive context-awareness discreet context-awareness.

The functionality of GeoHealth can be divided into four overall themes: (1) location-based spatial view of the work domain, (2) location-based information push, (3) location-based ad hoc exchange of work tasks and (4) location-based alarms. These are described and illustrated in detail in the subsections below.

\subsection{Location-based spatial view of the work domain}

The primary functionality of GeoHealth is to provide the distributed healthcare workers with a spatial view of their work domain. This is done by plotting the locations of patients, healthcare workers (tracked via GPS) and their planned tasks/routes for the day on a full-screen map of the municipality (Figure 1). The map can be navigated by means of dragging it left/right and up/down. Zooming and toggling between map view, satellite view and hybrid view (satellite images with superimposed map information) is done through on-screen controls. By clicking on a patient on the map, a call-out box appears with access to all the information available about this particular person (which used to be distributed on different paper-based records) and with functionality for taking notes (Figure 3). Through this box, it is also possible for the nurses to make changes to the medical records, treatment, etc. Changes and new information are immediately available to coworkers. Clicking on a telephone number listed in the patient records initiates a SkypeOut call. By clicking on a coworker on the map, it is possible to initiate a Skype call to this person's laptop.

\subsection{Location-based information push}

In addition to facilitating information 'pull' about the work domain through the map representation and call-out boxes, the system also 'pushes' information on the basis of location and assigned work tasks. However, rather than prompting the user with 
(a)



(c)

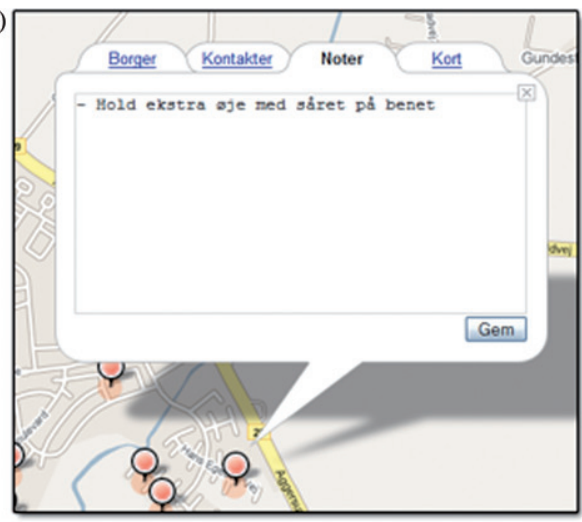

(b)

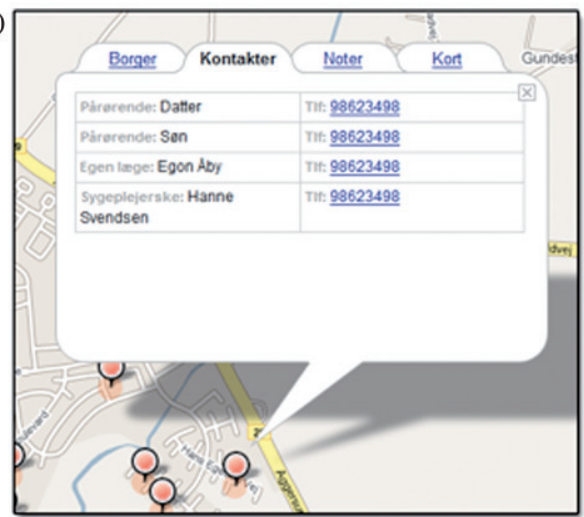

(d)



Figure 3. Four different views of patient information: (a) medical record, (b) contact information, (c) notes on treatment and (d) satellite image and map details of home location.

intrusive information screens, information push is done discreetly through gentle changes to the map representation in the form of appearance (and disappearance) of information. First, the map view moves automatically when the user comes close to the edges (as in a sat-nav system), and icons change automatically in accordance to coworkers progress throughout the day. Second, when a healthcare worker drives into the vicinity of an assigned patient, the call-out box for this patient automatically pops up on the map, making information about the upcoming visit ready at hand (Figure 4). In addition to electronic patient information, one of the tabs contains a close-up satellite image of the patient's address facilitating way finding through reference to landmarks and physical attributes of the environment (e.g. the colour of roofs, density of houses, presence of trees, etc.). When the healthcare worker leaves vicinity of a patient, the box automatically disappears again.

\subsection{Location-based ad hoc exchange of work tasks}

Apart from facilitating reshuffling of ones' own work tasks, the GeoHealth prototype system also facilitates easy ad hoc exchange of work tasks between 

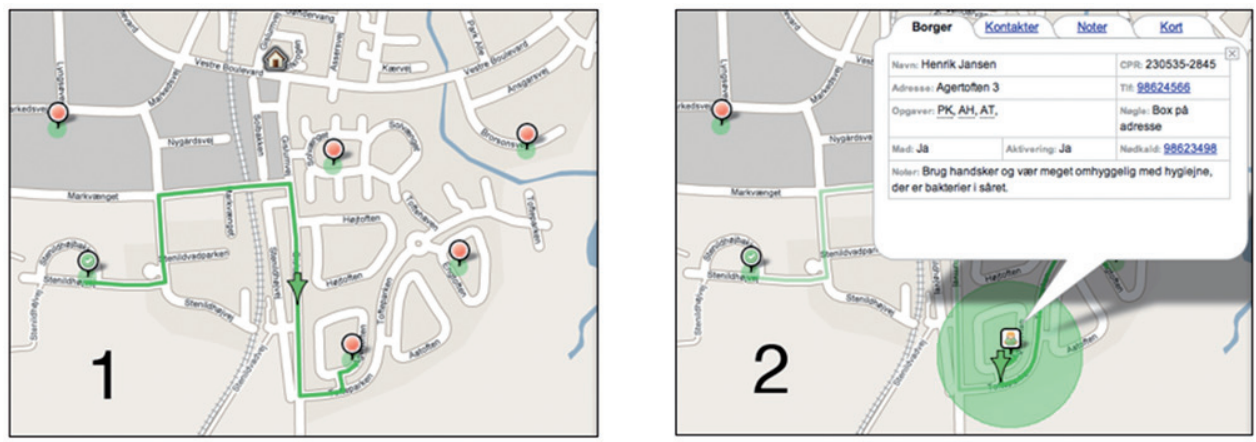

Figure 4. Information box automatically appearing when driving into vicinity of an assigned patient's home.



Figure 5. Location-based ad hoc exchange of work tasks by dragging a patient icon onto the location of another healthcare worker.

healthcare workers during a workday based on the location of coworkers. Negotiation of changes to the work plan is done through the systems' built-in text-messaging functionality or verbally through a Skype call, and the actual change to the work plan is done by simply dragging a patient icon onto the location of another healthcare worker on the map and then dropping it there (Figure 5). By doing this, the visit to that particular patient will disappear from one healthcare worker's list of tasks and appear on the other's. Moving a work task on to a coworker also triggers a pre-defined text notification to appear on the receiving person's screen. Hence, rather than forcing the users through tedious formalised procedures for negotiation and confirmation of changes to the work schedule (which happens all the time), this design relies on trust, professionalism and social 
conventions for collaboration. The representation of the location of patients and coworkers on the map assists the healthcare workers in assessing whom it would be best to hand over a particular task to.

\subsection{Location-based alarms}

For the purpose of replacing the use of mobile and landline phones for negotiating responds to alarms, and for supporting better decisions regarding such responses, GeoHealth embeds alarms directly into the spatial view of the work domain, and uses the continuous GPS positioning of the healthcare workers to automatically direct alarms to the nearest person. Hence when an alarm is triggered, the closest healthcare assistant or nurse receives a pop-up message on their screen, and the location of the alarm, as well as the location of two nearby colleagues, is highlighted on the map (Figure 6). If assistance is needed, the nearby colleagues can then be contacted via Skype. In the event that the closest healthcare worker is not able to respond, he or she can reject the alarm by which it is directed to the second closest person, and so forth. Again, this implementation represents a design decision to rely on the ability of the spatial representation of the shared work domain, in conjunction with the healthcare workers' professionalism, mutual trust and well-established social conventions, to inform well-functioning collaboration rather than enforcing more rules and formalisations on peoples' workday.

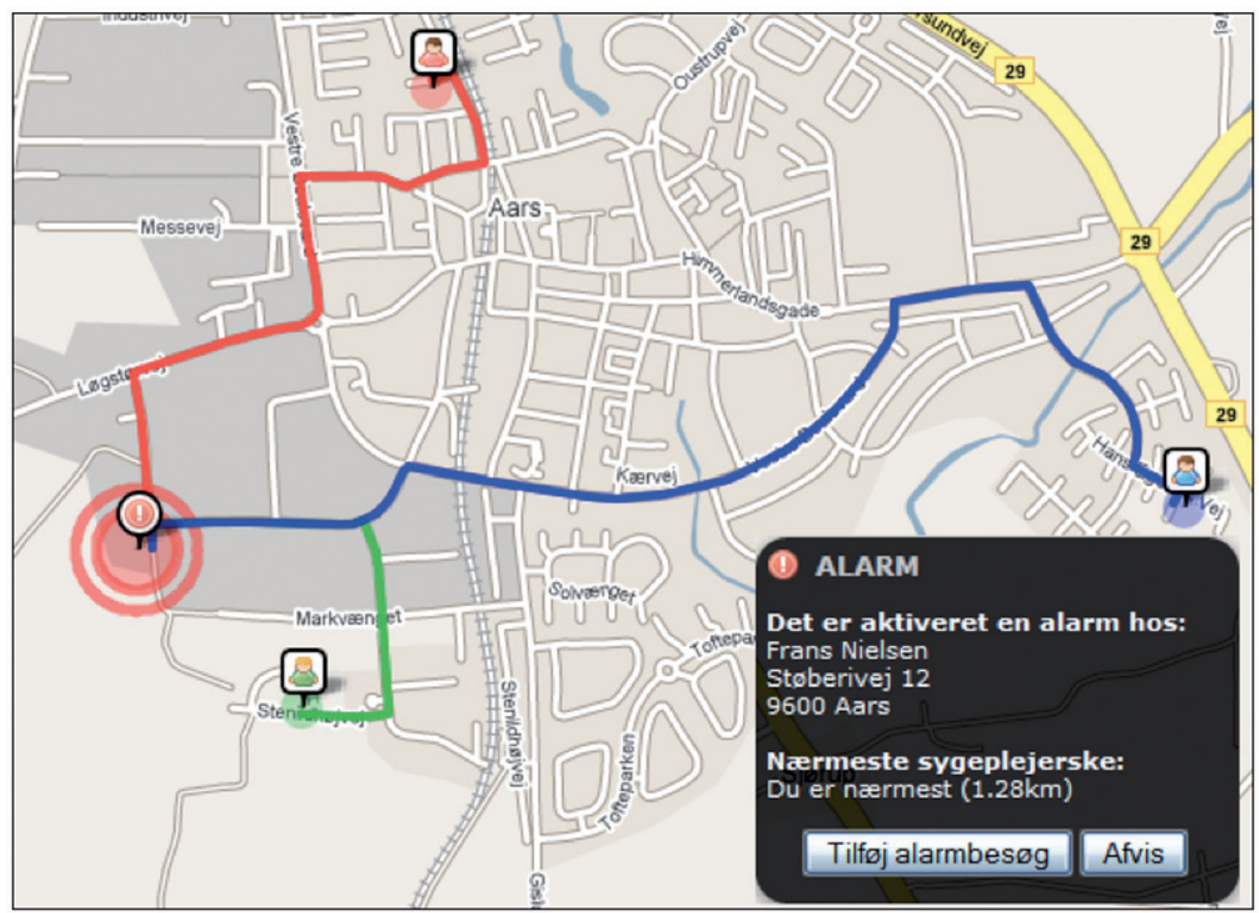

Figure 6. Patient alarm appearing as a pop-up message on the closest healthcare worker's screen along with information about location of the two most nearby colleagues. 


\subsection{Implementation}

GeoHealth was implemented using web technologies and languages such as XHTML (W3C 2002), CSS (W3C 2009), JavaScript (Eich 2005) and the Ajax framework (Garrett 2005), thus allowing the healthcare workers to interact with the system through a standard web browser. The server-side component was implemented on an experimental set-up consisting of a Debian Linux system, an Apache web server, a MySQL database server and the PHP scripting language engine (Lerdorf et al. 2002). For the evaluation studies, the GeoHealth prototype was accessed through a Firefox browser on an Apple MacBook connected to the Internet via general packer radio service (GPRS) on a Bluetooth-enabled mobile phone. The user's position was tracked with a GPS device connected to the MacBook's USB port.

GeoHealth's user interface is built on top of Google Maps embedded into a web page in full screen. The floating palettes are made from layered XHTML dynamically updated using JavaScript. The engine used to run the interface was created using object-oriented JavaScript and asynchronous XML requests, which makes the user interface independent of page reloading and improve possibilities for interactivity and system responsiveness to user interaction both locally and remote. In popular terms, Ajax provides a framework that allows developers to create desktop-like applications for the web by relying on XML for data transport 'behind the scenes' of the graphical interface. Thereby the user is able to interact with the application while it is requesting or processing data in the background even though the application is run in a web browser. The prototype architecture is illustrated in Figure 7.

The information layers superimposed onto the geographical map are constructed from markers and polylines. Locations of patients on the map are calculated on the basis of their addresses' latitude and longitude coordinates provided through a look-up in the address web service (AWS) provided by The National Survey and Cadastre of Denmark, which contains the geographical coordinates of all addresses in the country. Routes between addresses on the map are generated on the basis of a look-up in the Google Maps directions service, which return a keyhole markup language $(\mathrm{KML})$ file with a sequence of coordinates making up a correct path following the road network. All information requests to AWS and Google Maps directions are done through the Ajax engine. The information flow is illustrated in Figure 8.

Below is an example extract from a KML file defining a point on the route (one of several) and the geometry of the complete route from start to end point.

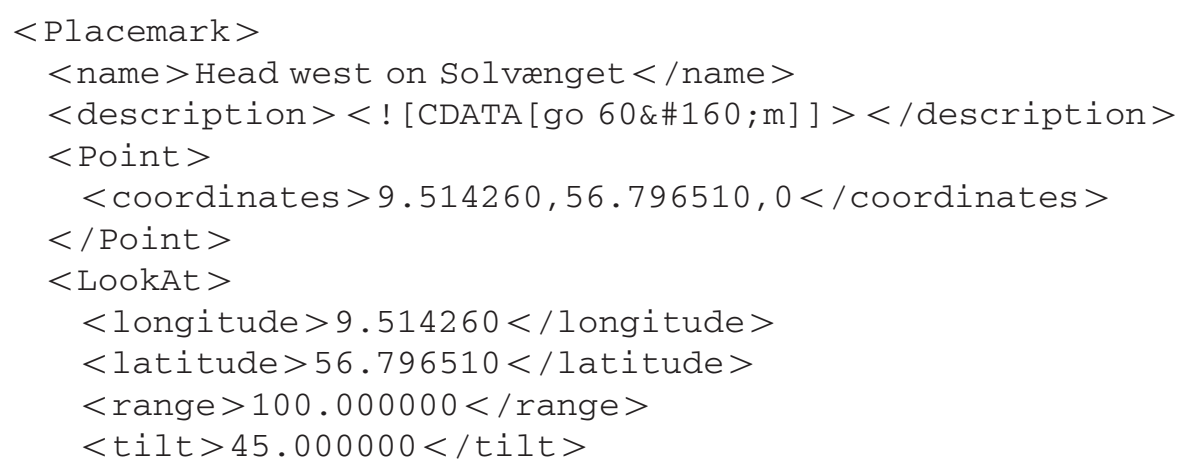




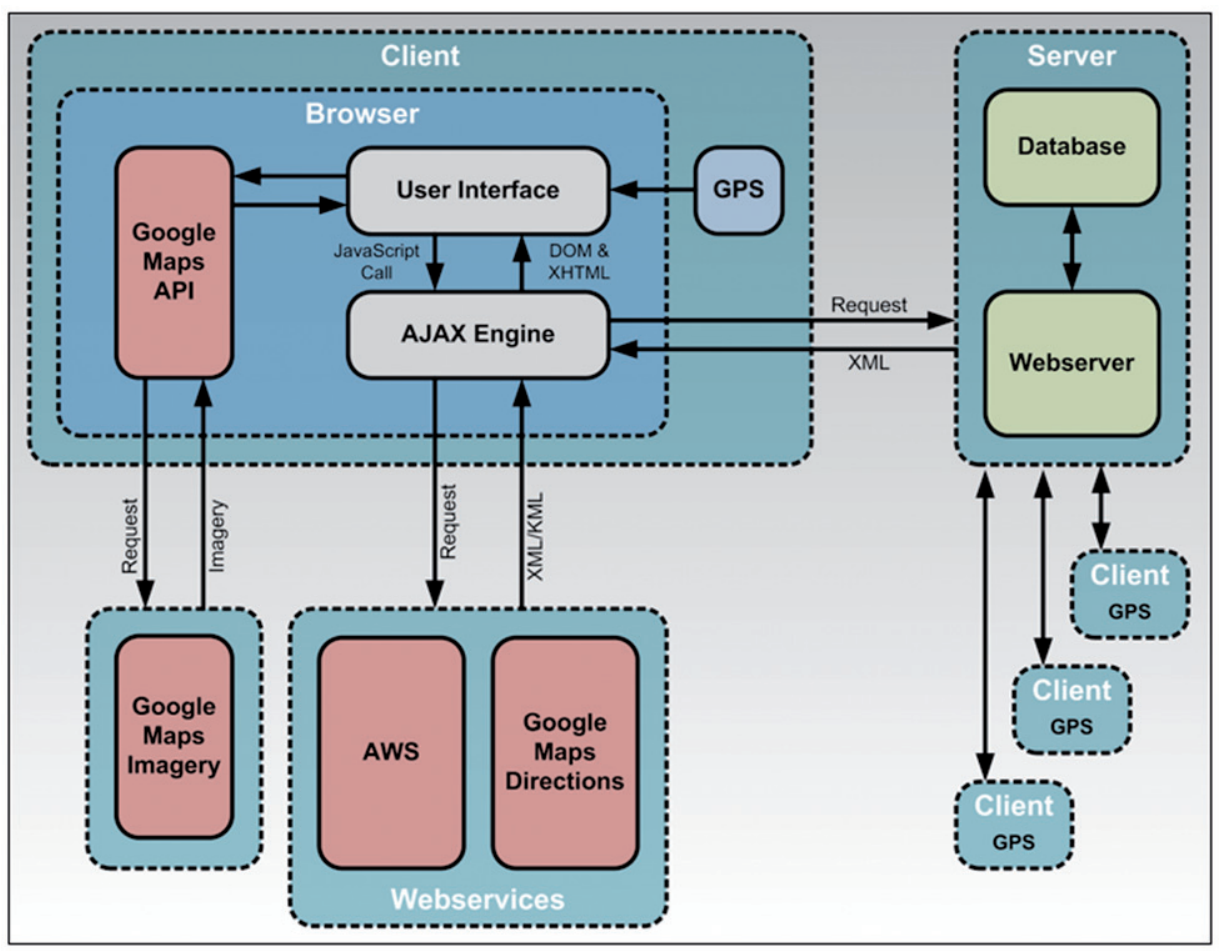

Figure 7. Technical architecture of the prototype system.

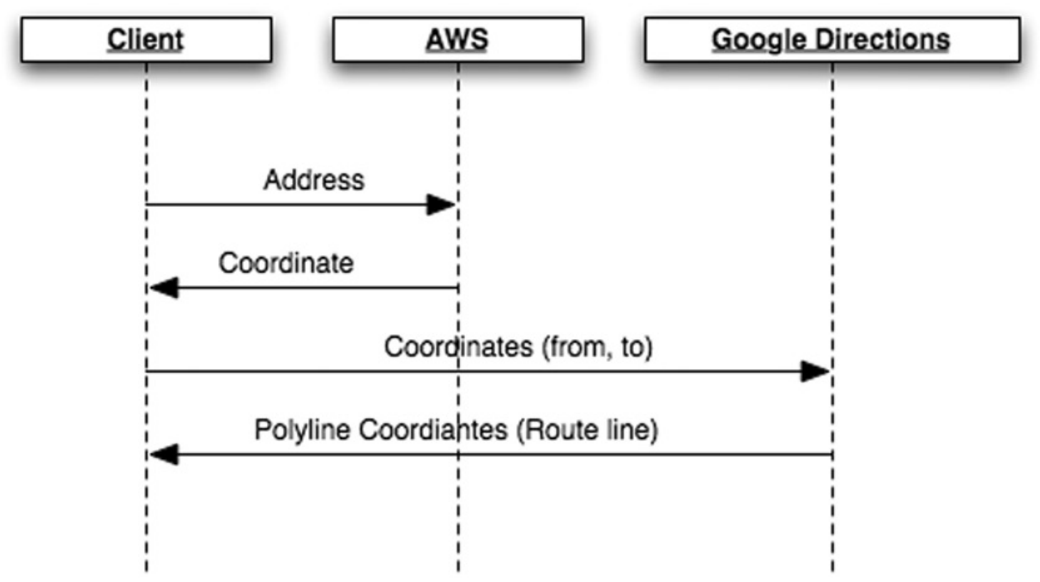

Figure 8. Information flow in the prototype system.

$<$ heading $>350.060608</$ heading $>$

$</$ LookAt $>$

$</$ Placemark $>$

(...)

$<\mathrm{Placemark}>$ 


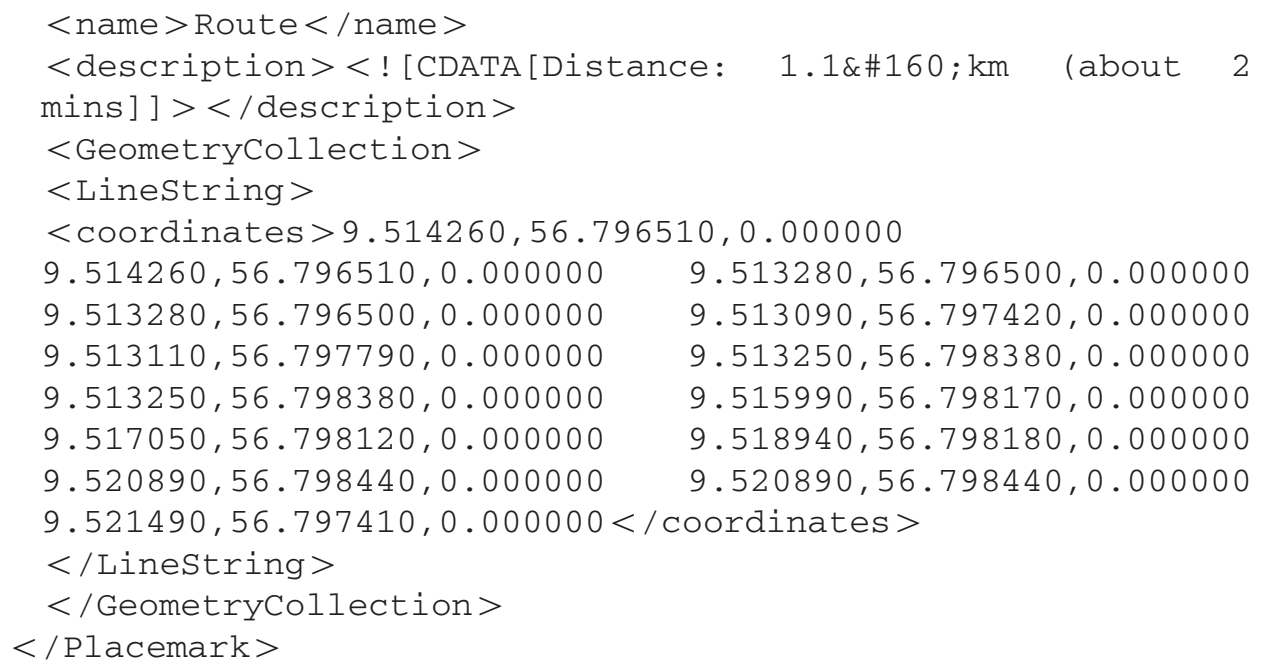

The individual client parts of the application running on the healthcare workers' laptops communicate with each other via a central database and a web server. However, unlike a conventional web site or web application, all requests to the web server are done through the Ajax engine and are requests for XML data rather than pages of XHTML. Hence, the content displayed in the browser is dynamically updated whenever content changes, and pages are not reloaded every time the user interacts with the system. As a result, the user experience of GeoHealth is much more smooth and continuous than that of conventional web applications.

Applications based on Google Maps are quite demanding on download bandwidth for graphics. For a map of $1700 \times 1200$ pixels, each zoom level requires $200 \mathrm{~Kb}-1 \mathrm{Mb}$ of data depending on view mode (map, satellite or hybrid). Since bandwidth on the mobile Internet is still a rather scarce and expensive resource in areas covered only by GPRS or 3G networks, the GeoHealth prototype system caches all data from the Google Maps imagery server locally on the client through a proxy server set-up. Thereby, maps and satellite images are only downloaded to the client once (until updated on the server), and the response time of the application has improved significantly. Using this approach, a GPRS connection of $56 \mathrm{kbit} / \mathrm{s}$ proved sufficient enough for running the prototype application smoothly once maps and satellite images have been cached. Although areas with cheap high-speed wireless broadband access will unquestionably grow, we believe that it is important to construct mobile Internet services and applications in a way that is still as bandwidth efficient as possible.

As GeoHealth is a network application with distributed clients, it relies on network connectivity to function optimally. In case of a network failure, some of the functionalities of the system would be lost, notably the ability to communicate and the exchange of real-time information. In relation to this, we subscribe to the principle of volatility promoted by Kindberg and Fox (2002). It is important to avoid designing systems on the misleading assumption that such failures are only rare. Instead, system designs should assume failure, and explicitly allow a graceful degrading of functionality in response without expensive processes of recovery. In the case of GeoHealth, using a caching approach as described earlier, the work tasks 
of each individual worker, and associated information from the central database, can be downloaded and stored locally on the client, while within in network coverage, thus minimising the implications of a network outage on the work activities. In case of a positioning failure, all information remains accessible by clicking on the map.

\section{Evaluations}

We evaluated GeoHealth through qualitative field and laboratory studies involving nine healthcare workers. The evaluations took place in the municipality of Aars, Denmark. The evaluation sessions were structured by task assignments prompting the users to interact with particular parts of the prototype. During the evaluation sessions, the users were asked to think aloud and respond to a series of interview questions about their perception, interaction and use of the system.

The aim of the field evaluation was to investigate into the use of the prototype system in realistic surroundings. For this purpose, we installed a laptop computer with GPS in a minibus equipped with video and audio recording equipment (Figure 9), and then let the healthcare workers use this vehicle to visit locations on their normal routes. As we were not at this point interested in issues related to interacting with the system while driving, most interaction took place while the vehicle was not in motion. The time used to drive from location to location was spent on follow-up questions and general discussions about the use of the system. The aim of the laboratory evaluations was to supplement findings from the field evaluation with more empirical data about the healthcare workers' perception and user experience of the prototype system. For this purpose, we set up a temporary

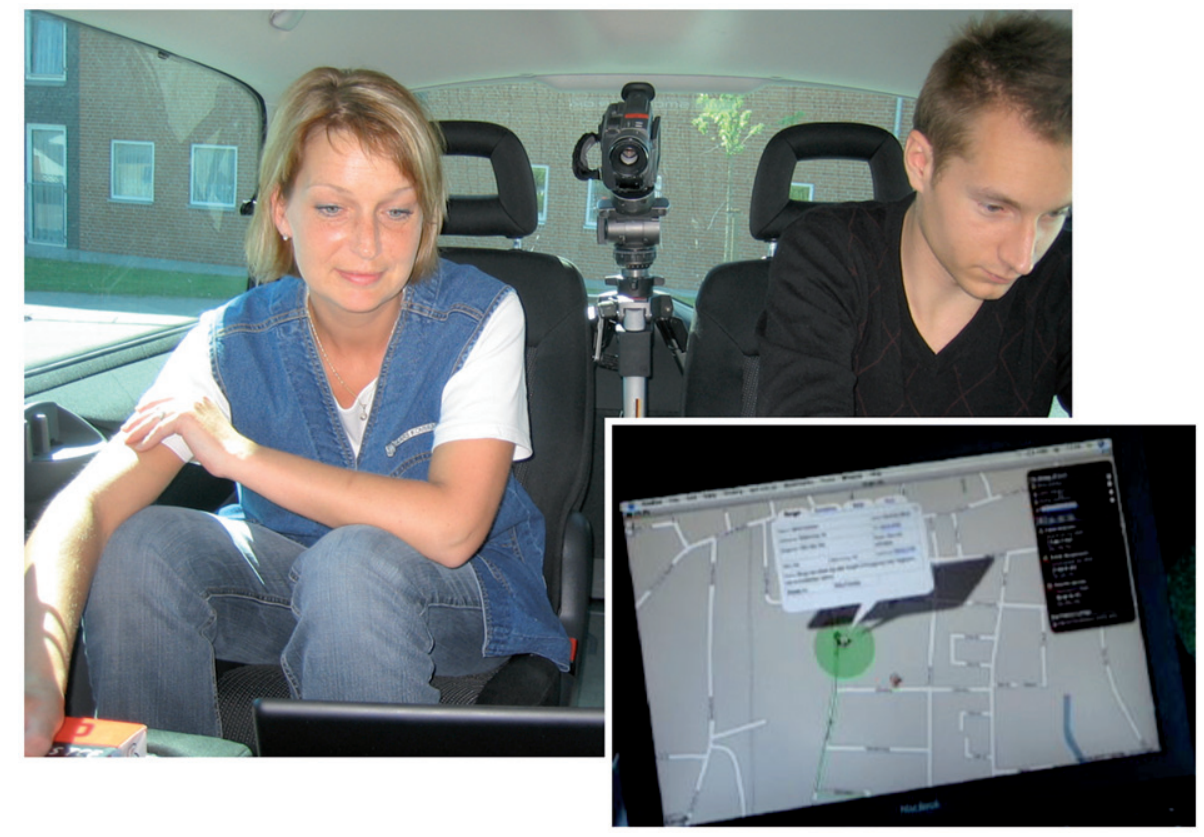

Figure 9. In-car field evaluation of GeoHealth prototype. 
laboratory in a dedicated room at the healthcare workers' central office, in which they could interact with the prototype system while seated at a desk.

The nine participating healthcare workers were all women and either employed as nurses or healthcare assistants. They were between 32 and 53 years old, and had 6 to 28 years of experience with their job. Most were experienced users of IT but one user characterised herself as a novice and one as an expert. All evaluations were recorded on digital video showing the users and their interactions with the system. In addition, the prototype screen was captured to a movie file using a screen logging utility running on the laptop. The evaluation sessions in the field took $30-40 \mathrm{~min}$, while the sessions in the lab took 25-30 min. For ethical reasons, none of the evaluation sessions involved real patients or real patient data but was based on fictive data and scenarios informed by our ethnographic studies of the domain.

Video data from the evaluation sessions underwent qualitative content analysis (Strauss 1987) carried out by two researchers with experience in human-computer interaction and qualitative analysis methods. Following the prescribed method, video data were first analysed and coded by the two researchers independently. Second, the two lists of codes were merged into one coherent list. The final list amounted to 25 unique codes. These codes were subsequently affinity diagrammed (Beyer and Holtzblatt 1998) into themes on higher levels of abstractions leading to the creation of eight overall categories (Figure 10). Below, we present and discuss highlights from these.

\section{Findings and discussion}

From our evaluations, it was clear that the users understood the basic design of GeoHealth and were able to interact with its basic functionalities after very

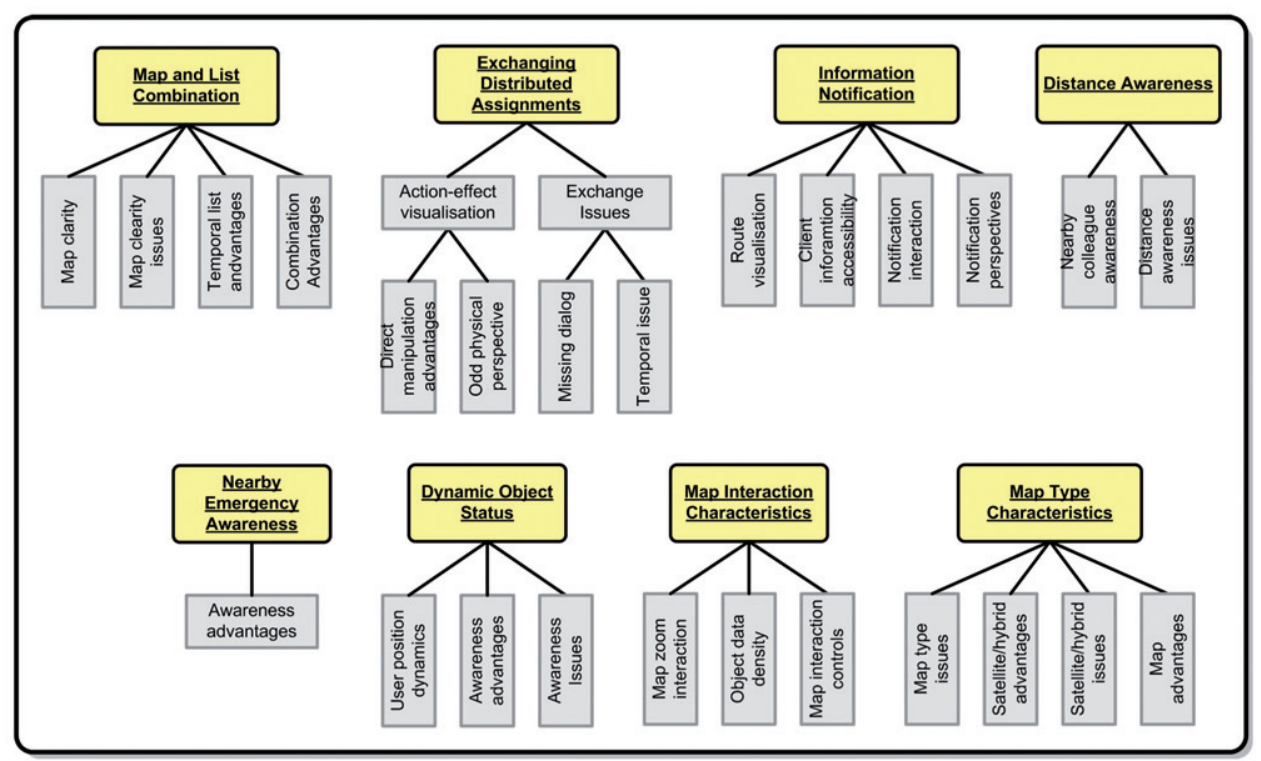

Figure 10. Thematic clustering of findings from the evaluations. 
short time. The users understood the map representations and the floating control panels (icons, colour coding, etc.), as well as the close relationship between them. They also expressed that the spatial and temporal/sequential representations of work tasks complemented each other very well, and that the spatial representation gave them a good and useful overview of their own work activities and supported awareness about their coworkers without requiring too much attention. On the downside, some concerns were voiced in relation to difficulty in separation of similar coloured routes and tasks on the map (i.e. the ones coloured red and the ones coloured orange). Obviously, this problem would increase if even more healthcare workers were to be represented at the same time, and great care should be taken when selecting a palette of possible colours. Some users also had problems with navigating and zooming the map.

Making information and note taking facilities about patients available electronically directly through call-outs on the map was received very positively, and it was confirmed that the use of satellite imagery aided navigation by means of prominent physical properties of the surroundings. However, it was also observed that the strong colours of satellite imagery sometimes interfered with the colour coding of work tasks and routes making the information overlays difficult to read (Denmark is a very green-looking country).

In relation to the location-based information push, the healthcare workers were very happy with the way GeoHealth automatically presented information about assigned patients when entering vicinity of their home. It was expressed that the change of visual appearance on the screen caught attention without being intrusive, and that it was 'natural' that the call-out box automatically disappeared when leaving vicinity. Nobody expressed, and it was not observed, that this use of context-awareness information push took control away from the user. However, concern was expressed that the status of a patient might change simply by driving past their house (i.e. from 'pending' to 'done'), and that such automatic change should require a certain 'dwell-time' at the physical location of the house. This concern confirms the issue of partitioning streams of location data highlighted by Raper et al. (2007), and addressed by, for example, Krimm and Horvitz (2006) and Liu et al. (2006).

In terms of support for location-based ad hoc exchange of work tasks, all healthcare workers were able to use the drag-and-drop technique with icons on the map. The simplicity of the procedure was appreciated, and users explicitly expressed that doing this within a spatial representation of the work domain helped them consider which colleague to negotiate exchanging tasks with based on vicinity to planned routes. On the negative side, however, some healthcare workers expressed that although they were able to understand and use the specific implemented interaction technique, it felt odd to move icons of patients to the healthcare workers on the map, since this did not match the corresponding effect in the real world (where the healthcare workers are the ones who go to the patients). As a solution, it was suggested to 'reverse the technique', so that one should drag the healthcare worker icons to the locations of the patients on the map instead in order to pair the two.

In relation to alarms, the healthcare workers were very positive towards the visualisation and automatic prompt of the closest person. Combined with the representation of nearby colleagues, it was expressed that this would significantly 
ease the amount of time as well as cognitive efforts spent on coordination. In extension of the new way of communicating in relation to alarms, the healthcare workers also found that the use of text-based messages constituted a significant advantage over current practice because it eased their need for making phone calls about every little detail. However, it was also stressed that text messages should not replace verbal communication, and that access to a verbal communication channel (e.g. Skype) would be essential for complex coordination as well as for the social purposes.

Extending the scope of patient monitoring beyond simple user-triggered alarms, it was discussed to embed live patient data into the GeoHealth system relayed from mobile wireless sensors over the Internet, as described in, for example, Figueredo and Dias (2004), Tachakra et al. (2003) and Varshney (2003).

As discussed in Raper et al. (2007, pp. 32-33), LBSs tracking the location of its users represent an ethical challenge of privacy and disclosure. In relation to this potential ethical issue of the GeoHealth application, none of the users expressed concerns about their movements during the workday being tracked via GPS. However, it should be noted that given the short time frame of the evaluations, this is not sufficient enough ground to conclude that tracking the location of mobile healthcare workers throughout their workday is acceptable as such. The ethical issues of this functionality should be investigated further, and mechanisms for ensuring privacy in this class of LBSs should be developed and evaluated with users.

\section{Conclusions and further work}

We have described an LBS, GeoHealth, for physically distributed and nomadic home healthcare workers that combine Google Maps, GPS positioning and Web 2.0 technology. The system provides information based on the user's location, represents live contextual data about a shared work domain on an interactive map displayed in a web browser, and has facilities for communicating and coordinating work tasks among distributed mobile users. Through user-based evaluations, we have verified the overall design and functionality of the prototype system as well as identified a number of areas for improvements to its functionality and interaction design.

From the user evaluations, we learned that the healthcare workers were positive towards the use of an LBS in their work activities while distributed throughout the municipality. Regarding the user experience of GeoHealth specifically, we learned that the dynamic and interactive map-based representation of the work domain was able to support collaboration, communication, coordination and peripheral awareness amongst the distributed coworkers. The users appreciated the overview of their shared work domain, the way information was gently pushed to them based on their location, the access to electronic patient data directly in the map view, the integration with voice- and text-based communication, and the location-based handling of alarms. On the basis of the feedback from our test users, we conclude that LBSs such as GeoHealth, built around Web 2.0 technologies, have unexploited potentials for the creation of new and positive user experiences within the domain of home healthcare. We speculate that similar LBSs would be applicable and valuable to other domains. 
The presented study leaves several areas for further work. First, the prototype should be extended into a fully functional and stable system with improved interaction design, which can be deployed into the home healthcare organisation for a longer period of time (e.g. 6-12 months). During this trial period, the prototype design should continuously be improved through iterative cycles of user feedback and changed to the interface design. Facilitated by this process, longitudinal studies of real-world use should be done in order to gain knowledge about long-term usage and potential changes happening to the way work is carried out in the use organisation triggered by the implementation of this new technology. As a part of this research, quantitative and qualitative measures should be done of improvements (or degradations) to the quality of service provided. Enquiries should also be made into the patients' perception of and opinion about the use of such technologies within home healthcare. Finally, the healthcare workers' perception of being tracked by GPS throughout their workday should be investigated.

For the first functional prototype iteration described in this article, our focus has been on the user experience of the functionality envisioned for GeoHealth. Hence, there are a number of technical aspects that we have not yet implemented, which should be dealt with in future iterations. First, the electronic patient record part was not integrated with the real national back-end database. Instead, a database was set up with temporary patient information for testing purposes. In order to do a realistic longitudinal study of GeoHealth, the information in the system should be integrated with the real electronic patient record for the municipality. Second, no mechanisms for dealing with issues of data security and privacy were implemented yet. Since GeoHealth handles sensitive and personal data, a fully implemented system, or even a prototype system deployed in the use organisation over a longer period of time, should, of course, protect data from unauthorised access, for example, by using encryption or secure protocols. Third, future implementation work should deal with the challenges of system scalability. Finally, in terms of network failure, it is important that the current network status and a potentially degraded state of operation (i.e. no real-time information updates) are communicated clearly to the user. The same goes for potential positioning failure.

GeoHealth was implemented in 2006 at a time where handheld computing platforms imposed several limitations on interaction designers and systems developers. One year later Apple released their iPod Touch and iPhone. These devices have significantly changed the landscape of mobile computing, and are much more powerful both in terms of system performance, mobile usability and flexibility to implement well-functioning mobile applications and, in particular, LBSs. Extending the work presented in this article, it would be highly interesting to design, implement and study the use of an iPhone-powered GeoHealth System. While much of the functionality of the presented prototype can be achieved technically on an iPhone, the question remains if the handheld form factor and limited screen real estate is sufficient or not for this type of application and in this particular use domain.

\section{Acknowledgements}

The authors thank the staff at the home healthcare unit of the municipality of Aars, Denmark, for their participation throughout the project. 


\section{References}

Aaltonen, A. and Lehikoinen, J., 2005. Refining visualization reference model for context information. Personal and Ubiquitous Computing, 9 (6), 381-394.

Bardram, J.E., 2004. Applications of context-aware computing in hospital work: examples and design principles. In: Proceedings of the 2004 ACM Symposium on Applied Computing SAC'04, 14-17 March 2004, Nicosia, Cyprus. New York, NY: ACM, 1574-1579.

Barkhuus, L. and Dey, A.K., 2003. Is context-awareness computing taking control away from the user? Three levels of interactivity examined. In: Proceedings of UbiComp 2003, Seattle, Washington, 149-156.

Beyer, H. and Holtzblatt, K., 1998. Contextual design. San Francisco: Morgan Kaufmann.

Boulos, M.N.K., 2003a. The use of interactive graphical maps for browsing medical health internet information resources. International Journal of Health Geographics, 2, 1.

Boulos, M.N.K., 2003b. Location-based information services: a new paradigm in personalised information delivery. International Journal of Health Geographics, 2, 2.

Boulos, M.N.K., et al., 2007. CAALYX: a new generation of location-based services in healthcare. International Journal of Health Geographics, 6 (9), 1-6.

Brown, B. and Randell, R., 2004. Building a context-sensitive telephone: some hopes and pitfalls for context sensitive computing. CSCW, 13 (3), 329-345.

Chang, A.Y., et al., 2009. Combining google earth and GIS mapping technologies in a dengue surveillance system for developing countries. International Journal of Health Geographics, 8, 49.

Cheverst, K., et al., 2000. Developing a context-aware electronic tourist guide: issues and experiences. In: Proceedings of CHI 2000, The Hague, The Netherlands, 20-31.

Dey, A. and Mankoff, J., 2005. Designing mediation for context-aware applications. ACM Transactions on Computer-Human Interaction, 12 (1), 53-80.

Driedger, S.M., et al., 2007. Correction: using participatory design to develop (public) health decision system support through GIS. International Journal of Health Geographics, 6, 53.

Edwards, W.K., 2005. Putting computing in context: an infrastructure to support extensible context-enhanced collaborative applications. ACM Transactions on Computer-Human Interaction, 12 (4), 446-474.

Eich, B., 2005. JavaScript at ten years. In: Proceedings of the tenth ACM SIGPLAN international conference on functional programming, Tallinn, Estonia. ACM, 129.

Erickson, T., et al., 2002. Social translucence: designing social infrastructures that make collective activity visible. Communications, 45 (4), 40-44.

Figueredo, M.V.M. and Dias, J.S., 2004. Mobile telemedicine system for home care and patient monitoring. In: Proceedings of the IEEE Engineering in Medicine and Biology Society (EMBS 2004), San Francisco, California, US, 3387-3390.

Garrett, J.J., 2005. Ajax: a new approach to web applications. Available from: http:// www.adaptivepath.com/ideas/essays/archives/000385.php [Accessed 6 September 2009].

Jones, Q., et al., 2004. People-to-people-to-geographical-places: the P3 framework for location-based community systems. CSCW, 13 (3), 249-282.

Kaasinen, E., 2003. User needs for location-aware mobile services. Personal and Ubiquitous Computing, 7 (1), 70-79.

Kindberg, T. and Fox, A., 2002. System software for ubiquitous computing. IEEE Pervasive Computing, 1 (1), 70-81.

Kjeldskov, J. and Paay, J., 2006. Public pervasive computing: making the invisible visible. IEEE Computer, 39 (9), 60-65.

Kjeldskov, J. and Skov, M.B., 2007. Exploring context-awareness for ubiquitous computing in the healthcare domain. Personal and Ubiquitous Computing, 11 (7), 549-562. 
Krimm, J. and Horvitz, E., 2006. Predestination: inferring destinations from partial trajectories. In: Proceedings of UbiComp 2006, Orange County, California, 243-260.

Lemmelä, S. and Korhonen, H., 2007. Finding communication hot spots of location-based postings. In: Proceedings of CHI 2007 work-in-progress, 2549-2554.

Lerdorf, R., et al., 2002. Programming PHP. Sebastopol, CA, USA: O’Reilly \& Associates Inc.

Liu, J., Wolfson, O. and Yin, H., 2006. Extracting semantic location from outdoor positioning systems. In: Proceedings of the 7th International Conference on Mobile Data Management (MDM'06), Nara, Japan. New York: IEEE Computer Society.

Maglogiannis, S. and Hadjiefthymiades, S., 2007. EmerLoc: location-based services for emergency medical incidents. International Journal of Medical Informatics, 76 (10), $747-759$.

Maglogiannis, I., et al., 2009. Enabling location privacy and medical data encryption in patient telemonitoring systems. IEEE Transactions on Information Technology in Biomedicine, 13 (6), 946-954.

Mandellos, G.J., et al., 2004. A novel mobile telemedicine system for ambulance yransport. Design and evaluation. In: Proceedings of the IEEE Engineering in Medicine and Biology Society (EMBS 2004), San Francisco, California, US, 3080-3083.

Mattila, E., et al., 2008. Mobile diary for wellness management - results on usage and usability in two user studies. IEEE Transactions on Information Technology in BioMedicine, 12 (4), 500-512.

Millen, D.R., 2000. Rapid ethnography: time deepening strategies for HCI field research. In: Proceedings of DIS 2000, New York City, New York, USA, 280-286.

Paay, J., 2008. From ethnography to interface design, In: J. Lumsden, ed. Handbook of research on mobile user interface design and evaluation. PA, USA: Idea Group, Inc., $1-15$, Chapter 1.

Paay, J., et al., 2009. Out on the town: a socio-physical approach to the design of a context aware urban guide. ACM Transactions on Computer-Human Interaction (TOCHI), 16 (2), 7-34.

Pattichis, C.S., et al., 2002. Wireless telemedicine systems: an overview. IEEE Antennas and Propagation Magazine, 44 (2), 143-153.

Paulos, E. and Goodman, E., 2004. The familiar stranger: anxiety, comfort and play in public places. In: Proceedings of CHI 2004, Vienna, Austria, 223-230.

Pitsillides, A., et al., 1999. DITIS: collaborative virtual medical team for home healthcare of cancer patients. In: Proceedings of Information Society and Telematics Applications, Catania, Italy.

Pruitt, J. and Adlin, T., 2006. The persona lifecycle: keeping people in mind throughout product design. San Francisco: Morgan Kaufmann.

Rainham, D., et al., 2008. Development of a wearable global positioning system forr place and health research. International Journal of Health Geographics, 7, 59.

Raper, J., et al., 2007. A critical evaluation of location based services and their potential. Journal of Location Based Services, 1 (1), 5-45.

Reponen, J., et al., 2000. MOMEDA - a Mobile smartphone terminal for DICOM images and web-based electronic patient data. Journal of Telemedicine and Telecare, 6 (1), 45-49.

Sawhney, N. and Schmandt, C., 2000. Nomadic radio: speech and audio interaction for contextual messaging in nomadic environments. ACM Transactions on ComputerHuman Interaction (TOCHI), 7 (3), 353-383.

Skov, M.B. and Høegh, R.T., 2006. Supporting information access in a hospital ward by a context-aware mobile electronic patient record. Personal and Ubiquitous Computing, 10 (4), 205-214.

Strauss, A., 1987. Qualitative analysis for social scientists. Cambridge: Cambridge University Press. 
Tachakra, S., et al., 2003. Mobile e-health: the unwired evolution of telemedicine. Telemedicine Journal and e-Health, 9 (3), 247-257.

Varshney, U., 2003. Pervasive healthcare. IEEE Computer, 36 (12), 138-140.

Woodward, B., Istepanian, R.S.H., and Richards, C.I., 2001. Design of a telemedicine system using a mobile telephone. IEEE Transactions on Information Technology in Biomedicine, $5(1), 13-15$.

W3C, 2002. XHTML ${ }^{\mathrm{TM}} 1.0$ The Extensible HyperText Markup Language (Second Edition) Available from: http://www.w3.org/TR/xhtml1/ [Accessed 6 September 2009].

W3C, 2009. Cascading Style Sheets Level 2 Revision 1 (CSS 2.1) Specification. Available from: http://www.w3.org/TR/CSS2/ [Accessed 6 September 2009]. 